\title{
a-convex convergent alternating series
}

\section{Hamsapriye}

To cite this article: Hamsapriye (2000) a-convex convergent alternating series, International Journal of Mathematical Education in Science and Technology, 31:5, 691-701, DOI:

10.1080/002073900434378

To link to this article: http://dx.doi.org/10.1080/002073900434378

\section{曲 Published online: 11 Nov 2010.}

Submit your article to this journal ๘

Џll Article views: 21

Q View related articles 5 
INT. J. MATH. EDUC. SCI. TEChNOL., 2000, vol. 31, NO. 5, 691-701

\title{
$\alpha$-convex convergent alternating series
}

\author{
HAMSAPRIYE \\ Department of Mathematics, Central College Campus, Bangalore University, Bangalore- \\ 560001, India. E-mail: preethi@bg1.vsnl.net.in
}

(Received 23 Fune 1999)

\begin{abstract}
Various types of convergent alternating series are studied and analysed. Certain important and interesting properties enjoyed by these classes of alternating series, pertaining to the range of the parameter $\alpha(0<\alpha<1)$, are discussed. A few illustrations are given, wherever necessary. Further, the generalized $\alpha$-convexity conditions, to be satisfied by the terms of a series which is $l$ times $\alpha$-convex, are discussed, along with the estimates of the remainders and the term to be added to any partial sum $S_{k}$ at any $l$ th stage of correction.
\end{abstract}

\section{Introduction}

In a recent work [1], Gal Ezer and $Z$ was described a correction procedure to sum a given alternating series of the type

$$
\sum_{n=0}^{\infty}(-1)^{n} a_{n} \quad a_{n}>0
$$

in which $a_{n+1} \leq a_{n}, \forall n$ and $a_{n} \longrightarrow 0$ as $n \longrightarrow \infty$. Such a correction procedure is applied to a chosen partial sum of the series (1.1) to obtain its sum more accurately. The correction procedure is carried out by introducing 'convexity conditions', which are satisfied by certain classes of alternating series. Briefly speaking, the convexity condition of [1] demands that the terms of the series (1.1), should satisfy the condition

$$
a_{n+1}-2 a_{n+2}+a_{n+3} \geq 0 \quad \forall n
$$

in which case the sum of the series can be estimated quite efficiently by adding the term

$$
\frac{1}{2}(-1)^{k+1} a_{k+1}
$$

to any chosen partial sum $S_{k}$, where we define

$$
S_{k}=a_{0}-a_{1}+a_{2}+\ldots+(-1)^{k} a_{k}
$$

The procedure for obtaining such corrected partial sums and corrected remainders can be utilized repeatedly, whenever additional covexity conditions hold for the terms of the series. This concept of convexity condition, which accelerates the summation procedure, has been generalized [2] to what are called 'generalized $\alpha$ convexity conditions'. We say that the series is once $\alpha$-convex if the terms of the series satisfy the following two conditions: 
and

$$
\left.\begin{array}{l}
(1-\alpha) a_{n+1}-a_{n+2}+\alpha a_{n+3} \geq 0 \\
\alpha a_{n+2}-a_{n+3}+(1-\alpha) a_{n+4} \geq 0
\end{array}\right\} \forall n
$$

In such a case the sum can be corrected by adding the term

$$
(-1)^{k+1} \alpha a_{k+1}
$$

to the partial sum $S_{k}$. Further corrections can be obtained, if the terms of the series satisfy the generalized $\alpha$-convexity conditions of the type (1.5) [2].

It may so happen that some classes of series violate the condition (1.2) and may possess different $\alpha$ s for $n$-even and $n$-odd separately. That is, a series may not be ' $\frac{1}{2}$-convex' in the sense of [1]. For example, series of the form:

$$
S^{ \pm}=\sum_{n=0}^{\infty}(-1)^{n}\left(\frac{a \pm(-1)^{n} b}{c^{n+1}}\right)
$$

with

$$
\begin{array}{lllll}
\text { (i) } a, b>0 & \text { (ii) } a>b & \text { (iii) } \quad c>1 & \text { (iv) } \quad c(a-b)>(a+b)
\end{array}
$$

violate the condition given in (1.2), whenever the relation

$$
\frac{a}{b}<\left(\frac{c+1}{c-1}\right)^{2}
$$

holds good. Such series can be effectively summed by imposing the generalized $\alpha$-convexity conditions. In fact, by imposition of the generalized $\alpha$-convexity conditions, we obtain the exact sum of the series at one stroke!! [2]. Therefore, by introducing the concept of generalized convexity conditions, we can sum a larger class of convergent alternating Leibnitz series.

In this paper, we prove some interesting properties enjoyed by the $\alpha$-convex and partially $\alpha$-convex series. We define a partially $\alpha$-convex series to be a series of the form (1.7), satisfying the conditions (1.8) and (1.9). In other words, such series are not $\frac{1}{2}$-convex. More precisely, they are not $\alpha$-convex for any $\alpha(0<\alpha<1)$, and they enjoy some interesting properties involving the range of $\alpha$. In section 2 , we state and prove the various properties of $\alpha$-convex and partially $\alpha$-convex series. Special examples are considered for clarity purposes. In section 3, we state the generalized $\alpha$-convex conditions to be satisfied by the terms of an $l$ times $\alpha$-convex series, considering the fact that if the conditions (1.5) are satisfied by a series then the series is said to be once $\alpha$-convex. We also establish estimates for the remainder term at any $(l-1)$ th stage and the term that we add at any $l$ th stage of correction.

\section{Important properties of the $\alpha$-convex series}

In this section, we state and prove some results, concerning the range of $\alpha$, for $\alpha$-convex alternating series and for partially $\alpha$-convex alternating series. We also examine a few examples to support our theoretical results.

Property 1. A convergent alternating series of the form (1.1) is once $\alpha$-convex if and only if it is $(1-\alpha)$-convex, for $0<\alpha<1$. 
Proof. We consider the series in (1.1), assuming that the terms of the series satisfy the $\alpha$ convexity conditions as stated in the relation (1.5). That is, we assume that the series is once $\alpha$-convex, for some $0<l \leq \alpha \leq m<1$. Thus, by interchanging the two relations, in (1.5), we obtain for any $\beta \in[l, m]$, that

and

$$
\left.\begin{array}{l}
\beta a_{n+2}-a_{n+3}+(1-\beta) a_{n+4} \geq 0 \\
(1-\beta) a_{n+1}-a_{n+2}+\beta a_{n+3} \geq 0
\end{array}\right\} \forall n
$$

Replacing $n$ by $(n-1)$ in the first inequality and by $n+1$ in the second inequality, we obtain

and

$$
\left.\begin{array}{l}
\beta a_{n+1}-a_{n+2}+(1-\beta) a_{n+3} \geq 0 \\
(1-\beta) a_{n+2}-a_{n+3}+\beta a_{n+4} \geq 0
\end{array}\right\} \forall n
$$

which may be re-written as

and

$$
\left.\begin{array}{l}
(1-(1-\beta)) a_{n+1}-a_{n+2}+(1-\beta) a_{n+3} \geq 0 \\
(1-\beta) a_{n+2}-a_{n+3}+(1-(1-\beta)) a_{n+4} \geq 0
\end{array}\right\} \forall n
$$

This shows that $(1-\beta) \in[l, m]$. The converse of the result can be proved on similar lines.

Example 1. We consider the series

$$
S=\sum_{n=0}^{\infty}(-1)^{n}\left(\frac{5-(-1)^{n}}{4^{n+1}}\right)
$$

It can be verified that the series is once $\alpha$-convex for $\alpha \in\left[\frac{1}{3}, \frac{2}{3}\right]$. It is also clear that Property 1 holds for the above example.

Property 2. Let the series of the form (1.7), along with the conditions as in (1.8), be once $\alpha$-convex. Suppose that $\alpha \in[p, q]$, for the case when $n$ is odd, then $\alpha \in[1-q, 1-p]$ for the case when $n$ is even.

Proof. It is proved in [2] that the series $S^{-}$of (1.7), along with the conditions (1.8), is once $\alpha$-convex, whenever

$$
p=\frac{c a-c b-a-b}{\left(c^{2}-1\right)(a+b)} \leq \alpha \leq \frac{c(c a-c b-a-b)}{\left(c^{2}-1\right)(a-b)}=q
$$

for $n$-odd and

$$
r=\frac{c a+c b-a+b}{\left(c^{2}-1\right)(a-b)} \leq \alpha \leq \frac{c(c a+c b-a+b)}{\left(c^{2}-1\right)(a+b)}=s
$$

for $n$-even. Using the above definitions of $p$ and $q$, it is straightforward to see that $r=1-q$ and $s=1-p$. A similar result holds for the series $S^{+}$, of (1.7). 
Example 2. We consider the series

$$
S=\sum_{n=0}^{\infty}(-1)^{n}\left(\frac{5-(-1)^{n} 2}{6^{n+1}}\right)
$$

It is easy to verify that [1] for the case when $n$ is odd we obtain

$$
p=\frac{11}{245} \leq \alpha \leq \frac{22}{35}=q
$$

and for $n$ even

$$
1-q=\frac{13}{35} \leq \alpha \leq \frac{234}{245}=1-p
$$

Property 3. For a partially convex series as stated by the relations (1.7), along with the conditions (1.8) and (1.9), the following statement is true:

If for $n$-odd, $\alpha \in[p, q]$ and for $n$-even $\alpha \in[r, s]$, then $[p, q] \cap[r, s]=\varnothing$.

Proof. A partially convex series violates the condition (1.2). In other words, the series is not $\frac{1}{2}$-convex. From property 2, we have for the case when $n$ is odd that $\alpha \in[p, q]$ and when $n$ is even $\alpha \in[1-q, 1-p] \equiv[r, s]$. Since the series is not $\frac{1}{2}-$ convex, $\frac{1}{2} \notin[p, q]$. Suppose that $p<q<\frac{1}{2}$, then $(1-p)>(1-q)>\frac{1}{2}$. In other words, $\frac{1}{2} \notin[1-q, 1-p]$. On the contrary, suppose that $\frac{1}{2}<p<q$, then $\left.\frac{1}{2}>1-p\right)>(1-q)$, or $\frac{1}{2} \notin[1-q, 1-p]$. Clearly, in both cases we see that $[p, q] \cap[r, s]=\varnothing$.

Example 3. We consider the example

$$
S^{-}=\sum_{n=1}^{\infty}(-1)^{n}\left(\frac{13-(-1)^{n} 3}{2^{n+5}}\right)
$$

For the case when $n$ is odd, we obtain $\frac{1}{12} \leq \alpha \leq \frac{4}{15}$ and for the case when $n$ is even we obtain $\frac{11}{15} \leq \alpha \leq \frac{11}{12}$. It is clear that the two intervals are disjoint. That is there is no common range for $\alpha$.

Property 4. Let the series given by the relation (1.7), along with the conditions (1.8) be once $\alpha$-convex. That is, for $\alpha \in[m, n]$ the terms of the series satisfy the conditions (1.5).

Then we have $m+n=1$.

Proof. From the relations (2.4) and (2.5) we have $p \leq \alpha \leq q$ for the case when $n$ odd and $r \leq \alpha \leq s$ for the case when $n$ even. From Property 2, we have $r=1-q$ and $s=1-p$. Again from property 1, we have that a series is once $\alpha$-convex if and only if it is once $(1-\alpha)$-convex, and so $0<p \leq \frac{1}{2}$ and $\frac{1}{2} \leq q<1$. Now if $p \leq 1-q$ then $q \leq 1-p$, and the common range for $\alpha$ is $[1-q, q]$. On the other hand, if $p \geq 1-q$ then $q \geq 1-p$, and the common range for $\alpha$ is $[p, 1-p]$. In either cases the sum of the numbers representing the two end-points is unity.

Example 4. We consider the series as stated in example 1. The series is once $\alpha$-convex for $\alpha \in\left[\frac{1}{3}, \frac{2}{3}\right]$ and $\frac{1}{3}+\frac{2}{3}=1$. 
Property 5. The series of the form

$$
S^{ \pm}=\sum_{n=0}^{\infty}(-1)^{n}\left(\frac{a \pm(-1)^{n} b}{(a+b)^{n+1}}\right), \text { with } a-b=1
$$

is not $\alpha$-convex, for any $\alpha \in(0,1)$.

Proof. By imposing the conditions (1.5) on the terms of the series $S^{-}$, we obtain $\alpha=0$ for $n$ odd and $\alpha=1$ for $n$ even. For the series $S^{+}$we obtain $\alpha=1$ for $n$ odd and $\alpha=0$ for $n$ even. This contradicts the fact that $\alpha \in(0,1)$.

Example 5. We consider the example of the series

$$
\sum_{n=0}^{\infty}(-1)^{n}\left(\frac{6+(-1)^{n} 5}{11^{n+1}}\right)
$$

Using the conditions (1.5), we obtain $\alpha=1$ when $n$ is odd and $\alpha=0$ when $n$ is even.

\section{Remarks}

(1) The range of $\alpha$ decreases with imposition of the $\alpha$-convexity conditions, at each stage.

(2) An alternating series may lose the convexity property after a finite number of stages.

In support of the remarks, we give a few more examples.

Example 6.

$$
\sum_{n=0}^{\infty}(-1)^{n}\left(\frac{4+(-1)^{n}}{3^{n+2}}\right)
$$

is once $\alpha$-convex for $\alpha=\frac{1}{2}$. We have observed that by imposing the condition (1.5), $\alpha \in\left[\frac{1}{2}, \frac{9}{10}\right]$, when $n$ is odd and $\alpha \in\left[\frac{1}{10}, \frac{1}{2}\right]$, when $n$ is even. Using the $\alpha$-convexity condition for the second time, that is [2]:

$$
\left.\begin{array}{l}
(1-\alpha)^{2} a_{n+1}-\left(1-\alpha+\alpha^{2}\right) a_{n+2}+\left(2 \alpha-\alpha^{2}\right) a_{n+3}-\alpha(1-\alpha) a_{n+4} \geq 0 \\
\text { and } \\
\qquad \alpha^{2} a_{n+2}-\left(1-\alpha+\alpha^{2}\right) a_{n+3}+\left(1-\alpha^{2}\right) a_{n+4}-\alpha(1-\alpha) a_{n+5} \geq 0
\end{array}\right\}
$$

on the terms of the above series, we see that for $n$ odd, $\alpha \in[0.6061072,0.75]$ and for the case where $n$ is even, $\alpha \in[0.25,0.3938928]$.

It is clear from the above example, that a series which is once $\alpha$-convex, need not be $\alpha$-convex for a second time. In other words, the range of $\alpha$ decreases as and when the $\alpha$-convexity conditions are imposed further. The above example shows that the series is $\frac{1}{2}$-convex once, but is not for the second time.

Example 7. We consider the example

$$
\sum_{n=0}^{\infty}(-1)^{n} \frac{1}{32^{n}}
$$


The above series is

$$
\begin{aligned}
& \text { once } \alpha \text { convex for } \alpha \in[0.030303,0.969696] \\
& \text { twice } \alpha \text { convex for } \alpha \in[0.161468,0.838532] \\
& \text { thrice } \alpha \text { convex for } \alpha \in[0.263576,0.736423] \\
& \text { fourth time } \alpha \text { convex for } \alpha \in[0.327513,0.672486]
\end{aligned}
$$

This is an example of an $\alpha$-convex alternating series which is four times $\frac{2}{3} \mathrm{rd}$ convex. It is also clear that the range of $\alpha$ decreases at each stage.

Property 6. Let a series of the form (1.1) be given. Let the series be once $\alpha$ convex, say, for $\alpha \in[p, q]$. Then, it is easy to prove that the range of $\alpha$, for the series $c \sum_{n=0}^{\infty}(-1)^{n} a_{n}$, for some $c>0$ is again $[p, q]$.

\section{Generalized $\alpha$-convexity conditions at the $l$ th stage}

We suppose that the alternating series

$$
\sum_{n=0}^{\infty}(-1)^{n} a_{n}, a_{n}>0
$$

is $\alpha$-convex, for some $\alpha \in(0,1)$, infinitely many times. In this section, we discuss the $\alpha$-convexity condition at any $l$ th stage. The major advantage of a series being $\alpha$-convex once, twice, thrice and so on, is that the sum of the series can be obtained more accurately, by adding certain correction terms to the partial sum $S_{k}$ of the series, mentioned earlier. We recall here that, if a series is once $\alpha$-convex, we add a term $(-1)^{k+1} \alpha a_{k+1}$ to $S_{k}$ [2] and obtain a corrected sum defined as

$$
\stackrel{\alpha}{\tilde{S}_{k}}=S_{k}+\alpha(-1)^{k+1} a_{k+1}
$$

and the remainder estimate is

$$
\left|\tilde{R}_{k}\right| \leq(1-\alpha)\left(a_{k+1}-a_{k+2}\right)
$$

Suppose the series is twice $\alpha$-convex, then the corrected sum in the second stage is $[2]$

$$
\stackrel{\alpha}{\tilde{S}_{k}}={\stackrel{\alpha}{S_{k}}}_{k}+\alpha(1-\alpha)(-1)^{k+1}\left(a_{k+1}-a_{k+2}\right)
$$

and the remainder estimate is

$$
\left|\stackrel{\alpha}{\tilde{\tilde{R}}_{k}}\right| \leq(1-\alpha)^{2} a_{k+1}-(1-\alpha) a_{k+2}+\alpha(1-\alpha) a_{k+3}
$$

In the third stage of the correction procedure, we have the corrected sum:

$$
\stackrel{\alpha}{\tilde{\tilde{S}}}_{k}={\stackrel{\alpha}{\tilde{S}_{k}}}_{k}+(-1)^{k+1} \alpha\left((1-\alpha)^{2} a_{k+1}-(1-\alpha) a_{k+2}+\alpha(1-\alpha) a_{k+3}\right)
$$

and the remainder estimate is 


$$
\begin{aligned}
\stackrel{\alpha}{\tilde{\tilde{R}}_{k}} \mid \leq & (1-\alpha)^{3} a_{k+1}-(1-\alpha)\left(1-\alpha+\alpha^{2}\right) a_{k+2}+(1-\alpha)\left(2 \alpha-\alpha^{2}\right) a_{k+3} \\
& -\alpha(1-\alpha)^{2} a_{k+4}
\end{aligned}
$$

and so on. The main motive of this section is to discuss the term that we add at any $l$ th stage, the remainder estimate and the $\alpha$-convexity conditions to be satisfied by the terms of the series, at that stage. Now the convexity conditions at the first and second stages are as given by the relations (1.5) and (2.7) respectively. The $\alpha$ convexity conditions to be satisfied by the terms of the series, if it is $\alpha$-convexity for the third time, are given below:

and

$$
\left.\begin{array}{c}
(1-\alpha)^{3} a_{n+1}-\left(1-2 \alpha+2 \alpha^{2}\right) a_{n+2}+\left(3 \alpha-4 \alpha^{2}+2 \alpha^{3}\right) a_{n+3} \\
-2 \alpha(1-\alpha) a_{n+4}+\alpha^{2}(1-\alpha) a_{n+5} \geq 0 \\
\alpha^{3} a_{n+2}-\left(1-2 \alpha+2 \alpha^{2}\right) a_{n+3}+(1-\alpha)\left(1+2 \alpha^{2}\right) a_{n+4} \\
-2 \alpha(1-\alpha) a_{n+5}+\alpha(1-\alpha)^{2} a_{n+6} \geq 0
\end{array}\right\}
$$

We have observed that the remainder estimate at the $(l-1)$ th stage is $(1-\alpha)$ times the first of the $\alpha$-convexity conditions at the $(l-2)$ th stage, replacing $n$ by $k$. Further, the first of the two convexity conditions at $(l-2)$ th stage can be obtained by dividing the expression that we add to $S_{k}$ at the $l$ th stage, by the factors $(-1)^{k+1} \alpha(1-\alpha)$ and then replacing $k$ by $n$, appending a ' $\geq 0$ ' symbol. The second of the convexity condition is obtainable from the first after replacing $\alpha$ by $(1-\alpha)$, and changing $n$ to $(n+1)$. Thus, if we can determine the expression to be added at the $(l+1)$ th stage, we can immediately derive the convexity conditions at the $(l-1)$ th stage and remainder estimate at the $l$ th stage.

Before we take up the general case, we first analyse the case for $\alpha=\frac{1}{2}$. Assuming that the given series is $\frac{1}{2}$-convex infinitely many times, we can correct the partial sum $S_{k}$, by adding the terms, given below, at various stages:

$$
\left.\begin{array}{ll}
\text { First stage } & (-1)^{k+1} \frac{1}{2} a_{k+1} \\
\text { Second stage } & (-1)^{k+1} \frac{1}{4}\left(a_{k+1}-a_{k+2}\right) \\
\text { Third stage } & (-1)^{k+1} \frac{1}{8}\left(a_{k+1}-2 a_{k+2}+a_{k+3}\right) \\
: & :
\end{array}\right\}
$$

It is easy to write down the term that we add at any $(l+1)$ th stage. Correspondingly, we can write down the convexity conditions satisfied by the terms of the series at that $(l-1)$ th stage and the remainder estimate at the $l$ th stage. It is interesting to note that, if the given series is $\frac{1}{2}$-convex infinitely many times and if we carry out the correction procedures of [1] infinitely many times, then we would add the entire remainder term $R_{k}$, to the chosen partial sum $S_{k}$. Thus, finally, we would obtain the exact sum of the series. This can be verified by adding all the terms corresponding to the relations (3.9). With this principle in mind, we have 
verified that in the case of generalized $\alpha$-convex series $(0<\alpha<1)$, the terms that we add are:

First stage $\quad(-1)^{k+1} \alpha a_{k+1}$

Second stage $(-1)^{k+1} \alpha(1-\alpha)\left[a_{k+1}-a_{k+2}\right]$

Third stage $\quad(-1)^{k+1} \alpha(1-\alpha)\left[(1-\alpha) a_{k+1}-a_{k+2}+\alpha a_{k+3}\right]$

Fourth stage $(-1)^{k+1} \alpha(1-\alpha)\left[(1-\alpha)^{2} a_{k+1}-\left(1-\alpha+\alpha^{2}\right) a_{k+2}\right.$

$$
\left.+\left(2 \alpha-\alpha^{2}\right) a_{k+3}-\alpha(1-\alpha) a_{k+4}\right]
$$

Fifth stage $\quad(-1)^{k+1} \alpha(1-\alpha)\left[(1-\alpha)^{3} a_{k+1}-\left(1-2 \alpha+2 \alpha^{2}\right) a_{k+2}\right.$

$\begin{array}{ll} & \left.+\left(3 \alpha-4 \alpha^{2}+2 \alpha^{3}\right) a_{k+3}-2 \alpha(1-\alpha) a_{k+4}+\alpha^{2}(1-\alpha) a_{k+5}\right] \\ \text { Sixth stage } \quad & -1)^{k+1} \alpha(1-\alpha)\left[(1-\alpha)^{4} a_{k+1}-\left(1-3 \alpha+4 \alpha^{2}-2 \alpha^{3}+\alpha^{4}\right) a_{k+2}\right. \\ & +\alpha\left(4-9 \alpha+8 \alpha^{2}-2 \alpha^{3}\right) a_{k+3}-\alpha(1-\alpha)\left(3-2 \alpha+2 \alpha^{2}\right) a_{k+4} \\ & \left.+\alpha^{2}(1-\alpha)(3-\alpha) a_{k+5}-\alpha^{2}(1-\alpha)^{2} a_{k+6}\right]\end{array}$

provided the series is $\alpha$-convex, as many times as required. Assuming that the series is $\alpha$-convex infinitely many times, we see that the sum of all the terms that we add to $S_{k}$ (i.e. the terms as in the relation (3.10)), yields the entire $R_{k}$, as is also observed in the particular case when $\alpha=\frac{1}{2}$. For a proof see the appendix.

\subsection{The term we add at any lth stage of correction}

A general formula for the term we add at any $l$ th stage, $(l \in \mathbb{N})$ is observed to be:

$$
\begin{aligned}
& (-1)^{k+1}\left[\alpha\left\{(1-(1-\alpha))^{-1}\right\}_{l} a_{k+1}\right. \\
& -\alpha(1-\alpha)\left\{(1-\alpha)^{-1}(1-(1-\alpha))^{-1}\right\}_{l-1} a_{k+2} \\
& +\alpha^{2}(1-\alpha)\left\{(1-\alpha)^{-1}(1-(1-\alpha))^{-2}\right\}_{l-2} a_{k+3}-\ldots \\
& \left.(-1)^{l-1} \alpha^{\left[\frac{l}{2}\right]}(1-\alpha)^{\left(\frac{l}{2}\right)}\left\{(1-\alpha)^{-\left(\frac{l}{2}\right)}(1-(1-\alpha))^{-\left[\frac{l}{2}\right]}\right\}_{1} a_{k+l}\right]
\end{aligned}
$$

where the symbols $\left(\frac{l}{2}\right)$ represents the integer less than or equal to $\frac{l}{2}$ and $\left[\frac{l}{2}\right]$ represents the integer greater than or equal to $\frac{l}{2}$. Also the notation \{\}$_{l}$ stands for the $l$ th polynomial expression, in $\alpha$, in the expansion of the term present in the braces. For instance, if we have to obtain $\left\{(1-\alpha)^{-1}(1-(1-\alpha))^{-2}\right\}_{3}$, then

$$
\begin{aligned}
\left\{(1-\alpha)^{-1}(1-(1-\alpha))^{-2}\right\}_{3}= & \left\{\left(1+\alpha+\alpha^{2}+\ldots\right)\left(1+2(1-\alpha)+3(1-\alpha)^{2}+\ldots\right)\right\}_{3} \\
= & \left\{1+[\alpha+2(1-\alpha)]+\left[\alpha^{2}+2 \alpha(1-\alpha)\right.\right. \\
& \left.\left.+3(1-\alpha)^{2}\right]+\ldots\right\}_{3} \\
= & {\left[\alpha^{2}+2 \alpha(1-\alpha)+3(1-\alpha)^{2}\right] } \\
= & 3-4 \alpha+2 \alpha^{2}
\end{aligned}
$$

Thus, in the fifth stage of the correction procedure (i.e. $l=5$ ). The coefficient of $a_{k+3}$, in the term we add to $S_{k}$ is

$$
\alpha^{2}(1-\alpha)\left\{(1-\alpha)^{-1}(1-(1-\alpha))^{-2}\right\}_{3}=\alpha(1-\alpha)\left(3 \alpha-4 \alpha^{2}+2 \alpha^{3}\right)
$$


Now based on the previous arguments, we give below the convexity conditions at the $(l-2)$ th stage and the remainder estimate at the $(l-1)$ th stage.

3.2. The convexity conditions at any $(l-2)$ th stage

$$
\left.\begin{array}{ll}
\frac{1}{\alpha(1-\alpha)} \quad\left[\alpha\left\{(1-(1-\alpha))^{-1}\right\}_{l} a_{n+1}\right. \\
& -\alpha(1-\alpha)\left\{(1-\alpha)^{-1}(1-(1-\alpha))^{-1}\right\}_{l-1} a_{n+2} \\
& +\alpha^{2}(1-\alpha)\left\{(1-\alpha)^{-1}(1-(1-\alpha))^{-2}\right\}_{l-2} a_{n+3}-\ldots \\
& \left.+(-1)^{l-1} \alpha^{\left[\frac{l}{2}\right]}(1-\alpha)^{\left(\frac{l}{2}\right)}\left\{(1-\alpha)^{-\left(\frac{l}{2}\right)}(1-(1-\alpha))^{-\left[\frac{l}{2}\right]}\right\}_{1} a_{n+l}\right] \geq 0
\end{array}\right\}
$$

for all $n \in N$.

\subsection{The Remainder estimate at any $(l-1)$ th stage}

$$
\begin{aligned}
\tilde{\check{\tilde{\tilde{R}}}}_{k} \mid \leq & \left.\left\{(1-(1-\alpha))^{-1}\right\}_{l} a_{k+1}-(1-\alpha)\{1-\alpha)^{-1}(1-(1-\alpha))^{-1}\right\}_{l-1} a_{k+2} \\
& +\alpha(1-\alpha)\left\{(1-\alpha)^{-1}(1-(1-\alpha))^{-2}\right\}_{l-2} a_{k+3}-\ldots \\
& +(-1)^{l-1} \alpha^{\left[\frac{l}{2}\right]-1}(1-\alpha)^{\left(\frac{l}{2}\right)}\left\{(1-\alpha)^{-\left(\frac{l}{2}\right)}(1-(1-\alpha))^{-\left[\frac{l}{2}\right]}\right\}_{1} a_{k+l}
\end{aligned}
$$

The expression (3.11) can be written in a more compact form as

$$
\mathcal{S}_{l}^{k}=\sum_{m=1}^{l}(-1)^{k+m} \alpha^{\left[\frac{m}{2}\right]}(1-\alpha)^{\left(\frac{m}{2}\right)}\left\{(1-\alpha)^{-\left(\frac{m}{2}\right)}(1-(1-\alpha))^{-\left[\frac{m}{2}\right]}\right\}_{l-m+1} a_{k+m}
$$

and we state that

$$
\sum_{l=1}^{\infty} \mathcal{S}_{l}^{k}=R_{k}
$$

We have obtained the results (3.11), (3.14) and (3.15) by studying the emergence of the patterns and we have not attempted to supply proofs.

\section{Conclusions}

We have analysed the properties of convergent alternating series of various types, such as $\alpha$-convex series and partially $\alpha$-convex series. The interesting properties enjoyed by these series pertaining to the parameter $\alpha(0<\alpha<1)$ are described. Certain examples are also worked out, in support of the various properties. Further, the generalized $\alpha$-convexity conditions at any $(l-2)$ th stage, the remainder estimate at any $(l-1)$ th stage and the expression that we 
add at any $l$ th stage, for correcting the partial sum $S_{k}$ are discussed and closed analytic expressions are given. We have also proved that if the correction procedure is carried out indefinitely, then indeed the remainder term $R_{k}$ gets added to the $k$ th partial sum $S_{k}$.

\section{Acknowledgements}

I wish to thank Professor A. Chakrabarti for his constant encouragement and for providing me with all the computing facilities.

I also wish to thank the referee for suggestions and comments which have improved the presentation of the paper considerably.

\section{Appendix}

Result

$$
\sum_{l=1}^{\infty} \mathcal{S}_{l}^{k}=R_{k}
$$

Proof. The term that we add at the $l$ th stage is (equation (3.16)):

$$
\begin{aligned}
\mathcal{S}_{l}^{k}= & \sum_{m=1}^{l}(-1)^{k+m} a_{k+m} \alpha^{\left[\frac{m}{2}\right]}(1-\alpha)^{\left(\frac{m}{2}\right)}\left\{(1-\alpha)^{-\left(\frac{m}{2}\right)}(1-(1-\alpha))^{-\left[\frac{m}{2}\right]}\right\}_{l-m+1} \\
= & (-1)^{k+1} a_{k+1} \alpha\left\{(1-(1-\alpha))^{-1}\right\}_{l} \\
& +(-1)^{k+2} a_{k+2} \alpha(1-\alpha)\left\{(1-\alpha)^{-1}(1-(1-\alpha))^{-1}\right\}_{l-1} \\
& +(-1)^{k+3} a_{k+3} \alpha^{2}(1-\alpha)\left\{(1-\alpha)^{-1}(1-(1-\alpha))^{-2}\right\}_{l-2}+\ldots \\
& +(-1)^{k+l} a_{k+l} \alpha^{\left[\frac{l}{2}\right]}(1-\alpha)^{\left(\frac{l}{2}\right)}\left\{(1-\alpha)^{\left(\frac{l}{2}\right)}(1-(1-\alpha))^{\left[\frac{l}{2}\right]}\right\}_{1}
\end{aligned}
$$

After $l$ stages we add the expression $\sum_{i=1}^{l} \mathcal{S}_{i}^{k}$ to the $k$ th partial sum $S_{k}$. Therefore, as $l \longrightarrow \infty$ we add the infinite series $\sum_{l=1}^{\infty} \mathcal{S}_{l}^{k}$. Thus,

$$
\begin{aligned}
\sum_{l=1}^{\infty} \mathcal{S}_{l}^{k}= & (-1)^{k+1} \alpha a_{k+1} \sum_{l=1}^{\infty}\left\{(1-(1-\alpha))^{-1}\right\}_{l} \\
& +(-1)^{k+2} \alpha(1-\alpha) a_{k+2} \sum_{l=2}^{\infty}\left\{(1-\alpha)^{-1}(1-(1-\alpha))^{-1}\right\}_{l-1} \\
& +(-1)^{k+3} \alpha^{2}(1-\alpha) a_{k+3} \sum_{l=3}^{\infty}\left\{(1-\alpha)^{-1}(1-(1-\alpha))^{-2}\right\}_{l-2}+\ldots \\
= & (-1)^{k+1} \alpha a_{k+1}(1-(1-\alpha))^{-1}
\end{aligned}
$$




$$
\begin{aligned}
& +(-1)^{k+2} \alpha(1-\alpha) a_{k+2}(1-\alpha)^{-1}(1-(1-\alpha))^{-1} \\
& +(-1)^{k+3} \alpha^{2}(1-\alpha) a_{k+3}(1-\alpha)^{-1}(1-(1-\alpha))^{-2}+\ldots \\
= & (-1)^{k+1} a_{k+1}+(-1)^{k+2} a_{k+2}+(-1)^{k+3} a_{k+3} \ldots \\
= & R_{k}
\end{aligned}
$$

Hence the result.

\section{References}

[1] Gal-Ezer, J., and Zwas, G., 1993, Int. F. Math. Educ. Sci. Technol., 24, 171-176.

[2] Chamrabarti, A., and Hamsapriye, 1996, Int. F. Math. Educ. Sci. Technol., 27, 565-582. 